\section{Validade e confiabilidade do índice de capacidade para o trabalho (ICT) em trabalhadores de enfermagem}

\author{
Validity and reliability of the work ability index \\ (WAI) in nurses' work
}

Escola Nacional de Saúde
Pública Sergio Arouca,
Fundação Oswaldo Cruz,
Rio de Janeiro, Brasil.
2 Instituto Oswaldo Cruz,
Fundação Oswaldo Cruz,
Rio de Janeiro, Brasil.
Correspondência
S. H. A. Silva Junior
Escola Nacional de Saúde
Pública Sergio Arouca,
Fundação Oswaldo Cruz.
Rua Julio Lins 109, Rio de
Janeiro, RJ 26330-260, Brasil.
sergio.edfisica@gmail.com

Abstract

This article assesses the validity and reliability of the work ability index (WAI) for 1,436 nurses in three public hospitals in Rio de Janeiro, Bra$z i l$. The study estimated the dimensional validity by means of exploratory factor analysis, as well as correlational validity, relating WAI to theoretically relevant constructs. Reliability was evaluated by internal consistency analysis, using Cronbach's alpha and McDonald's omega. Factor analysis indicated a two-dimensional structure explaining $52.8 \%$ of the accumulated variance for the principal components and $42 \%$ for the principal axes. The theoretical hypotheses for the construct's validity were confirmed, with direct and significant correlation between the WAI and the reward, control, and self-rated health scores; an inverse and significant correlation with the scale on need for recovery after work, minor psychological disorders, effort, over-commitment, and demand. In conclusion, the WAI showed adequate psychometric properties.

Health Personnel; Reproducibility of Results; Validity of Tests
Sérgio Henrique Almeida da Silva Junior 1 Ana Glória Godoi Vasconcelos 1

Rosane Harter Griep 2

Lúcia Rotenberg 2

\section{Introdução}

O índice de capacidade para o trabalho (ICT) é um instrumento que avalia a percepção do trabalhador em relação ao quão bem está, ou estará, neste momento ou num futuro próximo, e quão bem ele pode executar seu trabalho, em função das exigências, de seu estado de saúde e capacidades físicas e mentais ${ }^{1}$. Foi desenvolvido no contexto de envelhecimento da população mundial, com base na perspectiva de que promover a capacidade para o trabalho é uma forma de melhorar a qualidade do trabalho, a qualidade de vida e bem-estar, favorecendo uma aposentadoria ativa e com significado 2 . De fato, o ICT permite avaliar e detectar alterações, predizer a incidência de incapacidade precoce e subsidiar medidas preventivas de manutenção da saúde dos trabalhadores. É considerado um preditor de situações precoces de perda de capacidade laboral, aposentadorias precoces, absenteísmo por doença e desemprego ${ }^{3}$.

O ICT é construído com base em um questionário autoaplicável composto de dez itens, sintetizados em sete dimensões: (1) capacidade para o trabalho comparada com a melhor de toda vida, (2) capacidade para o trabalho em relação a exigências físicas, (3) número de doenças atuais diagnosticadas pelo médico, (4) perda estimada para o trabalho por causa de doenças, (5) faltas ao trabalho por doenças nos últimos 12 meses, (6) prognóstico próprio da capacidade para o 
trabalho daqui a 2 anos e (7) recursos mentais. $\mathrm{O}$ escore do ICT varia de 7 (pior índice) a 49 pontos (melhor índice). Sua aplicação no Brasil demanda como escolaridade mínima a quarta série do Ensino Fundamental 1. A versão em português e as instruções detalhadas para o cálculo do escore são encontradas em Tuomi et al. 1 .

No Brasil, os estudos sobre capacidade para o trabalho tiveram início na década de 90, após a tradução e adaptação do questionário para o português brasileiro, sendo utilizado em diversas pesquisas 4,5,6. A avaliação das propriedades psicométricas desses instrumentos constitui etapa fundamental que precede a investigação de associações envolvendo essas escalas e eventos em saúde, principalmente quando aplicadas em populações diversas daquelas nas quais foram desenvolvidas. Assim, estudos com amostras específicas como aqueles desenvolvidos com metalúrgicos 7 , eletricitários 8 e a utilizada no presente trabalho complementam os achados referentes às propriedades psicométricas, ainda escassos no Brasil 6.

Métodos estatísticos para avaliação de propriedades psicométricas têm sido propostos em função da importância atualmente atribuída à psicometria de instrumentos. Recente discussão sobre limitações do alfa de Cronbach como estimador de confiabilidade tem apontado outros métodos ainda pouco utilizados na literatura com propriedades mais desejáveis. Revelle \& Zinbarg ${ }^{9}$ realizaram um estudo comparando o desempenho de 13 estimadores de confiabilidade 10,11 e concluíram que o coeficiente ômega ( $\omega \mathrm{t})$ de McDonald apresentou melhor desempenho. Esse coeficiente baseia-se na proporção da variância comum em um teste e pode ser calculado utilizando-se a rotina desenvolvida no programa R (The R Foundation for Statistical Computing, Viena, Áustria; http://www.r-project.org), com base em análise fatorial exploratória.

Baseando-se nessas considerações, o presente trabalho pretende avaliar o desempenho psicométrico da versão brasileira do ICT no contexto de um estudo aplicado aos trabalhadores de enfermagem, por meio da comparação de diferentes técnicas na avaliação da confiabilidade e validade.

\section{Métodos}

A coleta de dados foi realizada em três hospitais públicos na cidade do Rio de Janeiro, entre junho de 2005 e março de 2006, com o objetivo de analisar as relações entre organização do trabalho hospitalar, a saúde e o bem-estar dos trabalhadores de enfermagem. Adotou-se a estratégia de apli- cação do questionário autopreenchível durante o horário de trabalho, após a leitura e assinatura do termo de consentimento, em locais reservados e com o apoio de aplicadores treinados.

O estudo teve base censitária, abarcando o conjunto de profissionais da assistência, que incluía enfermeiros, técnicos e auxiliares de enfermagem, independentemente do tipo de vínculo empregatício. De um total de 1.687 elegíveis, $1.509(89,4 \%)$ aderiram ao estudo. Desses, 4,8\% possuíam informações incompletas no preenchimento do ICT e por isto foram eliminados das análises do presente artigo, constituindo-se desta forma em 1.436 trabalhadores de enfermagem.

$\mathrm{O}$ instrumento multidimensional foi aperfeiçoado por meio de cinco rodadas de pré-testes. A versão final incluiu variáveis sociodemográficas, ocupacionais (categoria profissional, turno de trabalho, número de horas de trabalho profissional, número de empregos), hábitos relacionados ao estilo de vida (fumo, ingestão de bebidas alcoólicas, prática de atividade física) e diversas escalas em uso nas literaturas nacional e internacional: índice de capacidade para o trabalho 1 , estresse psicossocial do trabalho (escala de demanda-controle e desequilíbrio esforço-recompensa) 12,13, recuperação após o trabalho (escala de recuperação após o trabalho) 14, distúrbios psiquiátricos menores - Self-Report Questionnaire (SRQ-20) ${ }^{15}$. Informações mais detalhadas dos instrumentos utilizados no presente estudo encontram-se na Tabela 1.

\section{Procedimento de análise dos dados}

Testes de Kolmogorv-Smirnov foram realizados para verificar a aderência do escore do ICT à distribuição normal, determinando os tipos de testes estatísticos a serem utilizados. Em todas as análises o nível de significância foi de 5\%. Todos os cálculos foram feitos utilizando-se o programa R versão 9.0.1.

Os procedimentos de análise incluíram a avaliação da validade de construto (validades correlacional e dimensional) e estimativas de confiabilidade, que são detalhadas a seguir.

\section{- Validade de construto}

\section{a) Validade correlacional}

O construto capacidade para o trabalho é de natureza multifatorial por se originar do modelo estresse-desgaste 16 , que atribui o desgaste às cargas físicas e mentais do trabalho, ao ambiente, às ferramentas do trabalho e às características e recursos do próprio trabalhador 5 . Entre os fatores que atuam na redução da capacidade para o 
Hipóteses de validade de construto e características avaliadas.

\begin{tabular}{|c|c|c|c|}
\hline & Instrumento de avaliação & Descrição das características & Hipóteses avaliadas \\
\hline \multirow[t]{2}{*}{$\begin{array}{l}\text { Estresse psicossocial no } \\
\text { trabalho }\end{array}$} & $\begin{array}{l}\text { Versão brasileira da escala de } \\
\text { esforço, recompensa e excesso de } \\
\text { comprometimento } 31 \text {, adaptada do } \\
\text { questionário delineado por Siegrist } 13\end{array}$ & $\begin{array}{l}\text { Composto por três dimensões: (i) } \\
\text { esforço (demandas e obrigações), (ii) } \\
\text { recompensa (compensação em termos } \\
\text { de salário, segurança no trabalho, } \\
\text { perspectivas e estima) e (iii) excesso } \\
\text { de comprometimento (empenho } \\
\text { exagerado no trabalho, combinado a } \\
\text { um forte desejo de ser aprovado } \\
\text { e estimado) }\end{array}$ & $\begin{array}{l}\text { Escores mais elevados de esforço, mais } \\
\text { baixos de recompensa e mais altos de } \\
\text { excesso de comprometimento estão } \\
\text { associados a escores mais baixos de } \\
\text { capacidade para o trabalho }\end{array}$ \\
\hline & $\begin{array}{c}\text { Versão brasileira do Questionário } \\
\text { Sueco Demanda-Controle-Apoio } \\
\text { Social 13,32 }\end{array}$ & $\begin{array}{l}\text { Composto por duas escalas: (i) } \\
\text { demandas psicológicas (ritmo } \\
\text { e intensidade do trabalho) e (ii) } \\
\text { controle (autonomia e habilidade } \\
\text { requerida do trabalhador sobre o } \\
\text { processo). Atividades que envolvem } \\
\text { altas demandas psicológicas e baixo } \\
\text { controle favoreceriam o desgaste } \\
\text { no trabalho e adoecimento físico e } \\
\text { psicológico }\end{array}$ & $\begin{array}{l}\text { Escores mais elevados de demandas } \\
\text { psicológicas e escores mais baixos de } \\
\text { controle no trabalho estão associados } \\
\text { a escores mais baixos de capacidade } \\
\text { para o trabalho }\end{array}$ \\
\hline $\begin{array}{l}\text { Necessidade de se } \\
\text { recuperar do esforço } \\
\text { causado pelo trabalho }\end{array}$ & $\begin{array}{c}\text { Escala de necessidade de recuperação } \\
\text { após o trabalho, adaptada do } \\
\text { questionário descrito por van } \\
\text { Veldhoven \& Broersen } 33\end{array}$ & $\begin{array}{l}\text { Escala de } 11 \text { itens que avalia se } \\
\text { o tempo ou a possibilidade de } \\
\text { recuperação (durante ou após o } \\
\text { trabalho) é suficiente para repor o } \\
\text { esforço do trabalho. Em caso negativo, } \\
\text { há uma tendência a ocorrer um } \\
\text { processo acumulativo que, a longo } \\
\text { prazo, compromete a saúde } 14,34\end{array}$ & $\begin{array}{l}\text { Escores mais elevados de necessidade } \\
\text { de recuperação após o trabalho estão } \\
\text { associados a escores mais baixos de } \\
\text { capacidade para o trabalho }\end{array}$ \\
\hline Saúde física & Autopercepção de saúde 34,35 & $\begin{array}{l}\text { Item que avalia a autopercepção do } \\
\text { estado geral de saúde. É relacionado } \\
\text { com a mortalidade futura e fortemente } \\
\text { associado com o estado "real" ou } \\
\text { "objetivo" de saúde das pessoas 35,36 }\end{array}$ & $\begin{array}{l}\text { Indivíduos com pior percepção sobre } \\
\text { seu estado de saúde apresentam } \\
\text { escores mais baixos de capacidade } \\
\text { para o trabalho }\end{array}$ \\
\hline Saúde mental & $\begin{array}{l}\text { Self-Report Questionnaire } \\
\text { (SRQ-20), proposta por Harding } \\
\text { et al. } 36 \text { e validada no Brasil por } \\
\text { Mari \& Williams } 15\end{array}$ & $\begin{array}{c}\text { Escala composta por } 20 \text { itens, que } \\
\text { permite o rastreamento de transtornos } \\
\text { mentais comuns (depressão, } \\
\text { ansiedade, distúrbios somatoformes e } \\
\text { neurastenia) } 37\end{array}$ & $\begin{array}{c}\text { Indivíduos classificados com distúrbios } \\
\text { psíquicos menores apresentam escores } \\
\text { mais baixos de capacidade para o } \\
\text { trabalho }\end{array}$ \\
\hline
\end{tabular}

trabalho, aqueles relacionados à saúde são os de maior impacto 1 . Assim, variáveis ligadas à presença de sintomas e de doenças, à autopercepção de saúde e à saúde mental já foram associadas à capacidade para o trabalho 4,5,6. Fatores do ambiente psicossocial do trabalho também têm efeitos sobre a capacidade de trabalho ${ }^{5}$.

Com base no exposto, o estudo da validade de construto se baseou em variáveis ligadas à saúde (física e mental), à fadiga e aos fatores psicossociais do trabalho, como descrito na Tabela 1, que também apresenta as hipóteses testadas no presente estudo. Foram calculadas as correlações de Spearman (níveis de significância de 5\%) entre o ICT e diferentes escalas relacionadas ao estresse psicossocial no trabalho, recuperação após o trabalho e instrumentos de avaliação da saúde física e mental. 


\section{b) Validade dimensional}

Considerando que a maioria das variáveis que compõem o ICT são categóricas ordinais, exceto a variável "capacidade para o trabalho atual comparada com a melhor de toda a vida" considerada como contínua, optou-se por utilizar nas análises as correlações policóricas (entre variáveis ordinais) e biserial (variáveis ordinais e contínuas). Conforme recomendação de Tabachnick \& Fidell 17 , a fatorabilidade dos dados foi avaliada pelo índice de Kaiser-Meyer-Olkin (KMO), que indica a proporção da variância dos dados comuns a todas as variáveis, os seguintes critérios são adotados para avaliação desse teste: 1,0-0,9 muito bom; 0,9-0,8 bom; 0,8-0,7 médio; 0,7-0,6 razoável; 0,6-0,5 fraco; < 0,5 inaceitável. Também aplicou-se o teste de esfericidade de Bartlett que avalia se a matriz de correlação é uma matriz identidade, o que indicaria que não há correlação entre as variáveis. Valores significantes do teste $(p<0,05)$ implicam boa adequação dos dados à análise fatorial.

Para determinação do número de fatores a serem retidos utilizou-se análise paralela. Existem evidências segundo Horn 18 que esse é um método mais acurado para retenção de fatores 19 . Análise paralela consiste em gerar uma amostra aleatória randômica e comparar os autovalores da amostra empírica com os autovalores dessa amostra aleatória randômica. São retidos na análise os fatores que possuem um autovalor maior do que o autovalor correspondente da amostra aleatória randômica.

Procedeu-se à análise fatorial exploratória pelo método de extração eixos principais. Esse método é mais apropriado para explorar dimensões latentes com dados que não apresentam distribuição normal, sendo indicado por diferenciar a parte da variância comum da específica, ao contrário de componentes principais que não fazem esta distinção 19,20,21. Estipulou-se na interpretação do fator carga fatorial mínima de $\pm 0,3020$. Também foram calculadas as comunalidades referentes a cada item definidas como a parcela da variância do item explicada pelos fatores retidos na análise. Valores maiores ou iguais a 0,40 são considerados satisfatórios 21 .

Para rotação da matriz de cargas foram utilizados os processos oblíquo e ortogonal, buscando-se explorar a melhor configuração de cargas e interpretabilidade dos fatores, admitindo-se no primeiro caso que os fatores apresentam-se correlacionados. A correlação entre os fatores foi estimada no processo de rotação oblíquo, e sendo esta significante oferece uma forma de verificar a adequação do método de rotação. Também foi realizada a rotação ortogonal pelo método vari- max, no intuito de comparar o presente estudo com os que avaliaram as propriedades psicométricas do ICT.

\section{- Confiabilidade}

Neste estudo, a confiabilidade foi avaliada utilizando-se os índices alfa de Cronbach e o ômega $(\omega t)$ de McDonald disponíveis na rotina psych do $\mathrm{R}$ (psych: procedures for psychological, psychometric and personality research. http://cran. r-project.org/web/packages/psych/index.html).

A relação entre os estimadores alfa ( $\alpha$ ), ômega $(\omega t)$ e a confiabilidade ( $\rho$ ) de uma escala ou teste pode ser estabelecida usando-se a seguinte equação: $\alpha<\omega t<\rho$. O ômega fornece a estimativa mais próxima da confiabilidade e o alfa o menor valor 22 .

\section{Aspectos éticos}

O estudo foi aprovado pelos Comitês de Ética em Pesquisa da Fundação Oswaldo Cruz (Fiocruz) e dos hospitais envolvidos, e pela Comissão Nacional de Ética em Pesquisa (CONEP - Brasília), por envolver cooperação estrangeira.

\section{Resultados}

No presente estudo $(\mathrm{n}=1.436), 87,3 \%$ dos participantes eram mulheres, 57,1\% tinham Ensino Superior completo e a média de idade foi de 40 anos (desvio-padrão - DP = 12,7 anos), variando de 16 a 70 anos. Em relação às características ocupacionais, $28,4 \%$ eram enfermeiros, $20 \%$ técnicos e $51,6 \%$ auxiliares de enfermagem, $47,7 \%$ eram servidores públicos, $41,5 \%$ trabalhavam à noite e $37,9 \%$ referiram dois ou mais empregos na enfermagem.

O escore médio do ICT foi de 38,5 pontos ( $\mathrm{DP}=6,1$ pontos). Essa variável não apresentou aderência à distribuição normal $(\mathrm{p}<0,0001$ no teste de Kolmogorov-Smirnov), estabelecendo o uso de testes não paramétricos para realização das análises estatísticas.

No que se refere à validade de construto, o ICT mostrou-se correlacionado com alguns conceitos teoricamente relevantes. Apresentou correlação direta e significativa com os escores de recompensa (escala de desequilíbrio esforço-recompensa), controle (escala de demandacontrole) e autoavaliação do estado de saúde. Correlacionou-se inversamente com recuperação após o trabalho (escala de recuperação após o trabalho), distúrbios psíquicos menores (SRQ20), com as dimensões esforço e excesso de comprometimento (escala de desequilíbrio esforço- 
recompensa) e com o escore de demanda (escala de demanda-controle) (Tabela 2).

Os resultados do teste KMO $(0,82)$ e do teste de esfericidade de Bartlett $\left(\chi^{2}=3039,38 ; \mathrm{p}<\right.$ 0,001) mostraram a adequação dos dados para análise fatorial. A análise paralela indicou dois fatores para serem extraídos na análise fatorial, para os quais os autovalores obtidos com base nos dados atuais (Figura 1) apresentaram valores maiores do que os autovalores obtidos de forma aleatória. O critério de Kaiser apontou o mesmo número fatores. Portanto, as análises posteriores foram baseadas em dois fatores.

Os dois fatores extraídos explicam $42 \%$ da variância comum e 53\% da variância total dos dados (Tabela 3). Tanto as cargas quanto as comunalidades são relativamente maiores quando calculadas por componentes principais, com todos os itens apresentando valores de comunalidades acima de 0,40 por esse método. Considerando o ponto de corte 0,30 como a carga mínima para

\section{Tabela 2}

Coeficiente de correlação de Spearman entre o escore do índice de capacidade para o trabalho (ICT) e escore de construtos teoricamente relevantes $(n=1.436)$.

\section{Construtos teoricamente relevantes}

Correlação de Spearman

\begin{tabular}{lc}
\hline Recuperação após o trabalho & $-0,326$ * \\
Desequilíbrio esforço-recompensa & \\
Esforço & $-0,364$ * \\
Recompensa & 0,216 * \\
Excesso de comprometimento & $-0,271$ * \\
Escala de demanda-controle & \\
Demanda & $-0,212$ * \\
Controle & 0,179 * \\
Distúrbios psíquicos menores & $-0,456$ * \\
Autoavaliação do estado de saúde & 0,458 *
\end{tabular}

\section{Figura 1}

Autovalores dos dados atuais versus dados aleatórios.

Análise paralela

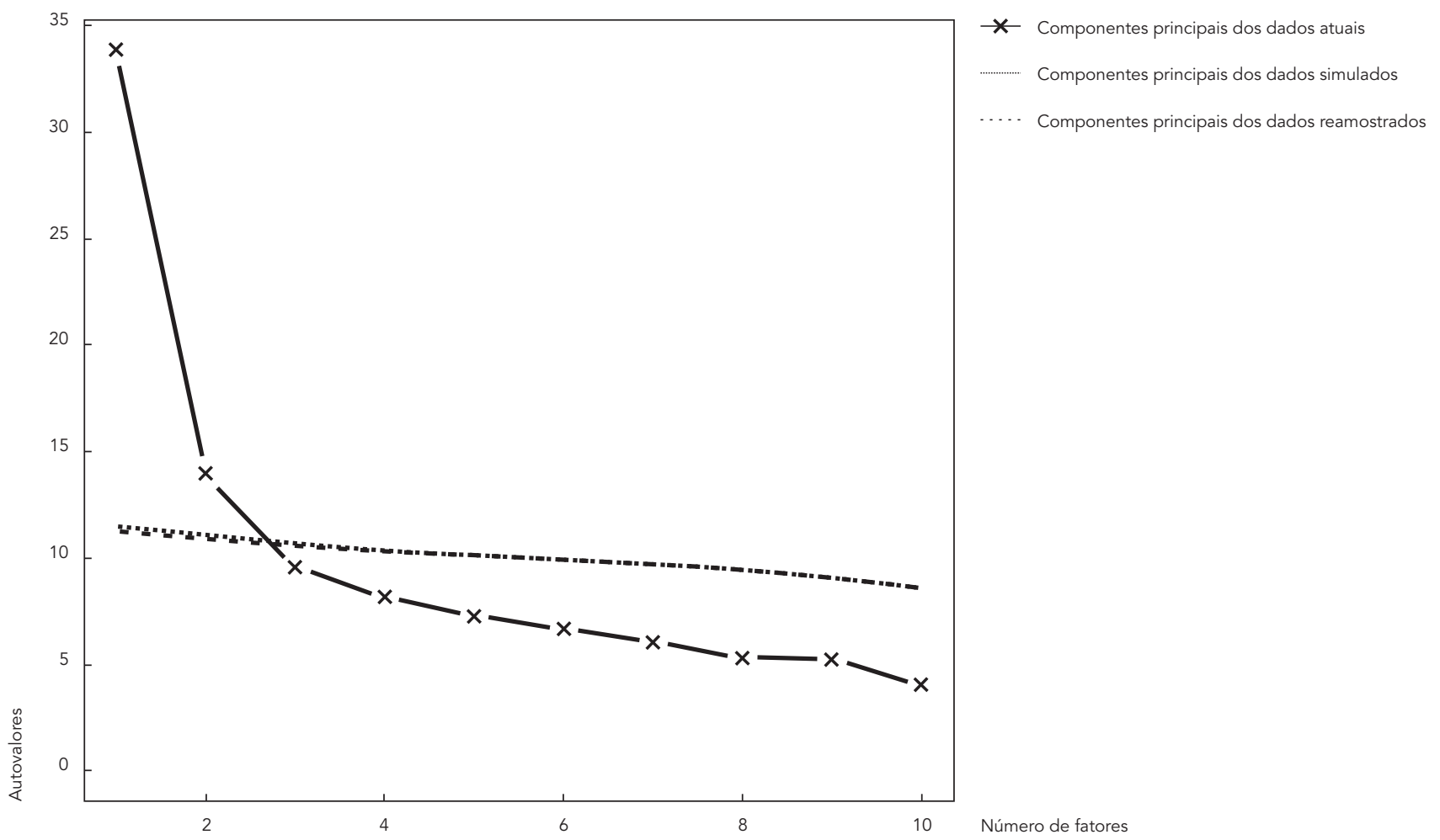


Tabela 3

Análise dos componentes principais e dos eixos principais do índice de capacidade para o trabalho (ICT), rotação varimax e utilizando a matriz de correlação policórica $(n=1.436)$.

\begin{tabular}{|c|c|c|c|c|c|c|c|}
\hline \multirow{2}{*}{$\begin{array}{l}\text { Dimen- } \\
\text { sões }\end{array}$} & \multirow[t]{2}{*}{ Itens } & \multicolumn{3}{|c|}{ Componentes principais } & \multicolumn{3}{|c|}{ Eixos principais } \\
\hline & & Fator 1 & Fator 2 & $h^{2}$ & Fator 1 & Fator 2 & $h^{2}$ \\
\hline 1 & $\begin{array}{l}\text { 1. Capacidade para o trabalho atual comparada com a } \\
\text { melhor de toda a vida }\end{array}$ & 0,61 & 0,22 & 0,43 & 0,52 & 0,25 & 0,33 \\
\hline \multirow[t]{2}{*}{2} & $\begin{array}{l}\text { 2. Capacidade para o trabalho em relação às exigências } \\
\text { físicas do trabalho }\end{array}$ & 0,51 & 0,51 & 0,52 & 0,47 & 0,48 & 0,45 \\
\hline & $\begin{array}{l}\text { 3. Capacidade para o trabalho em relação às exigências } \\
\text { mentais do trabalho }\end{array}$ & 0,67 & 0,12 & 0,46 & 0,55 & 0,17 & 0,33 \\
\hline 3 & 4. Número atual de doenças diagnosticadas pelo médico & 0,01 & 0,82 & 0,67 & 0,01 & 0,72 & 0,52 \\
\hline 4 & 5. Perda estimada para o trabalho devido às doenças & 0,26 & 0,77 & 0,66 & 0,27 & 0,72 & 0,58 \\
\hline 5 & 6. Faltas ao trabalho por doenças nos últimos 12 meses & 0,01 & 0,70 & 0,49 & 0,12 & 0,51 & 0,27 \\
\hline 6 & $\begin{array}{l}\text { 7. Prognóstico próprio sobre a capacidade para o trabalho } \\
\text { daqui a dois anos }\end{array}$ & 0,42 & 0,46 & 0,39 & 0,38 & 0,39 & 0,30 \\
\hline \multirow[t]{3}{*}{7} & 8. Apreciação das atividades diárias & 0,76 & 0,11 & 0,59 & 0,71 & 0,13 & 0,52 \\
\hline & 9. Sentimento de estar ativo e alerta & 0,78 & 0,01 & 0,62 & 0,74 & 0,15 & 0,55 \\
\hline & 10. Esperança para o futuro & 0,66 & 0,12 & 0,45 & 0,56 & 0,16 & 0,33 \\
\hline \multicolumn{2}{|c|}{ Variância do componente (\%) } & 30,0 & 23,0 & & 24,0 & 18,0 & \\
\hline \multicolumn{2}{|c|}{ Variância acumulada (\%) } & 30,0 & 53,0 & & 24,0 & 42,0 & \\
\hline
\end{tabular}

$h^{2}$ : comunalidade.

inclusão do item no fator, tem-se que o fator I reuniu os itens capacidade para o trabalho atual comparada com a melhor de toda a vida; capacidade para o trabalho em relação às exigências mentais do trabalho; apreciação das atividades diárias; sentimento de estar ativo; e alerta e esperança para o futuro, apresentando um autovalor de 3,387 e explicando $30 \%$ da variância total. O fator II foi composto por três itens: número atual de doenças diagnosticadas pelo médico; perda estimada para o trabalho devido às doenças; $\mathrm{e}$ faltas ao trabalho por doenças nos últimos 12 meses, com um autovalor de 1,39, explicando $23 \%$ da variância total. Os itens capacidade para o trabalho em relação às exigências físicas do trabalho e prognóstico próprio sobre a capacidade para o trabalho daqui a dois anos, tinham cargas fatoriais semelhantes e mais baixas nos dois fatores. A correlação estimada entre os dois fatores pelo método de rotação oblíquo é positiva e significante $(r=0,43 ; \mathrm{p}<0,001)$. No entanto, $o$ padrão de cargas é semelhante ao apresentado na rotação ortogonal, assim optou-se por mostrar apenas os resultados baseados nesta rotação (Tabela 3).

A Tabela 4 expressa a confiabilidade do ICT mensurada pelos estimadores alfa de Cronbach e ômega $(\omega \mathrm{t})$ de McDonald com base nas correlações biserial e policórica. $\mathrm{O}$ valor do alfa de
Cronbach e do ômega de McDonald foi, respectivamente, 0,80 e 0,87 , e a exclusão de qualquer questão não alterou substancialmente o resultado dos mesmos.

\section{Discussão}

O presente estudo investigou a versão brasileira do ICT no que concerne à validade e confiabilidade em trabalhadoras da enfermagem. Todas as hipóteses de associação em relação a aspectos da saúde do trabalhador e de seu ambiente de trabalho foram confirmadas por meio de correlações estaticamente significantes entre os escores do ICT e os construtos teoricamente relevantes. A análise dimensional identificou dois fatores que agregaram a maior parte dos itens, o que encontra respaldo em estudo anterior com equipes de enfermagem de dez países europeus ${ }^{23}$. A consistência interna apresentou melhor desempenho do que o observado em outras pesquisas com profissionais da enfermagem 23 ou outras categorias profissionais 8 .

Em relação à validade de construto, as correlações entre aspectos da saúde física e mental e o ICT mostram que aqueles com ICT mais elevado correspondem aos que avaliaram positivamente a própria saúde e referiram menos 
Valor do alfa de Cronbach e do omega( $\omega \mathrm{t})$ de McDonald por itens e para o escore global do índice de capacidade para o trabalho (ICT) em trabalhadores de enfermagem $(n=1.436)$.

\begin{tabular}{|c|c|c|}
\hline Itens & Alfa de Cronbach * & Omega de McDonald * \\
\hline 1. Capacidade para o trabalho comparada com a melhor de toda vida & 0,80 & 0,87 \\
\hline 2. Capacidade para o trabalho em relação às exigências físicas do trabalho & 0,79 & 0,86 \\
\hline 3. Capacidade para o trabalho em relação às exigências mentais do trabalho & 0,80 & 0,87 \\
\hline 4. Número de doenças atuais diagnosticadas pelo médico (pontos) & 0,81 & 0,87 \\
\hline 5. Perda estimada para o trabalho devido às doenças & 0,79 & 0,86 \\
\hline 6. Faltas ao trabalho por doenças nos últimos 12 meses & 0,81 & 0,88 \\
\hline 7. Prognóstico próprio da capacidade para o trabalho daqui a dois anos & 0,80 & 0,87 \\
\hline 8. Apreciação das atividades diárias & 0,79 & 0,86 \\
\hline 9. Sentir-se ativo e alerta & 0,79 & 0,86 \\
\hline 10. Esperança para o futuro & 0,80 & 0,87 \\
\hline Total & 0,80 & 0,87 \\
\hline
\end{tabular}

* Se a questão for excluída.

distúrbios psíquicos menores. Esses resultados confirmam os de Radkiewicz et al. 23 em relação a 38 mil trabalhadores de enfermagem. Achados semelhantes foram descritos por Martinez et al. 8, que encontraram associação positiva e estatisticamente significante entre os escores do ICT e de um instrumento genérico e multidimensional de avaliação da qualidade de vida, que engloba a saúde física e mental. No estudo desses autores, as dimensões de saúde física apresentaram correlações mais fortes com o ICT em comparação com a saúde mental, ao passo que na presente pesquisa as correlações foram semelhantes.

O conceito de capacidade para o trabalho é baseado na concepção da percepção individual de demandas no trabalho e o enfrentamento das mesmas. Nesse sentido, o ICT é um instrumento potencial para identificar riscos à saúde do trabalhador provenientes de um desequilíbrio entre a saúde, os recursos pessoais e as demandas no trabalho 24. Estudos epidemiológicos indicam a contribuição do estresse psicossocial no trabalho, mensurado por meio dos instrumentos de demanda-controle ou desequilíbrio esforço-recompensa na explicação dos efeitos adversos na saúde 25,26,27. Além disso, Bethge et al. 28 demonstraram o impacto desses estressores físicos e psicossociais na capacidade para o trabalho. Segundo os autores, o risco de uma limitada capacidade para o trabalho foi superior para pessoas com alta demanda e baixo controle no trabalho (odds ratio - OR = 5,62; intervalo de 95\% de confiança IC95\%: 3,55; 8,89) e com elevado desequilíbrio entre esforço e recompensa $(\mathrm{OR}=3,78$; IC95\%: $2,60 ; 5,51)$. O estudo também identificou que os trabalhadores com capacidade limitada para o trabalho percebem o seu ambiente de trabalho como particularmente estressante. No presente estudo, a correlação entre os baixos escores do ICT e a maior necessidade de recuperação após o trabalho remete à observação de Sluiter et al. 29, segundo a qual a necessidade de recuperação reflete os efeitos a curto prazo da carga de trabalho, que em sua repetição afetam a saúde dos trabalhadores e, neste sentido, tendem a reduzir a capacidade para o trabalho.

Pesquisas sobre a estrutura dimensional do ICT têm chegado a diferentes resultados. No Brasil, Martinez et al. 8 em estudo com trabalhadores do setor elétrico identificaram, por meio de componentes principais, três fatores com autovalores maiores do que 1 , explicando $57,9 \%$ da variância total $(20,6 \%, 18,9 \%$ e $18,4 \%$ para o 1o, 2 o e 3 o fatores, respectivamente). Radkiewicz et al. 23 investigaram a estrutura dimensional do ICT em profissionais de enfermagem e identificaram, utilizando-se de componentes principais, dois fatores explicando a estrutura dimensional do ICT em oito países, e apenas um fator em dois países.

No estudo de Martinez et al. 8, o primeiro fator agrega os itens apreciação das atividades diárias; sentimento de estar ativo e alerta; e esperança para o futuro que representa a dimensão "recursos mentais", o segundo fator é composto pelos itens capacidade para o trabalho atual comparada com a melhor de toda a vida; capacidade para o trabalho em relação às exigências físicas do trabalho; e capacidade para o trabalho em relação às exigências mentais do trabalho, que 
representa a percepção do trabalhador quanto à sua capacidade para o trabalho. $O$ terceiro fator reúne os itens: número atual de doenças diagnosticadas pelo médico; perda estimada para o trabalho devido às doenças; faltas ao trabalho por doenças nos últimos 12 meses; e prognóstico próprio sobre a capacidade para o trabalho daqui a dois anos, relativos à presença de doenças e restrições decorrentes do estado de saúde.

A estrutura dimensional do ICT neste estudo apresenta-se mais próxima da encontrada por Radkiewicz et al. 23 constituída de dois fatores, em que o primeiro foi formado dos itens capacidade para o trabalho atual comparada com a melhor de toda a vida; capacidade para o trabalho em relação às exigências físicas do trabalho; capacidade para o trabalho em relação às exigências mentais do trabalho; prognóstico próprio sobre a capacidade para o trabalho daqui a dois anos; apreciação das atividades diárias; sentimento de estar ativo e alerta; e esperança para o futuro, e o segundo fator os itens número atual de doenças diagnosticadas pelo médico; perda estimada para o trabalho devido às doenças; e faltas ao trabalho por doenças nos últimos 12 meses. Portanto, com exceção dos itens capacidade para o trabalho em relação às exigências físicas do trabalho; e prognóstico próprio sobre a capacidade para o trabalho daqui a dois anos que carregaram nos dois fatores neste estudo, os outros itens apresentaram padrão similar. Vale ressaltar que no estudo citado o item perda estimada para o trabalho devido às doenças apresentou cargas de magnitude semelhante nos dois fatores em sete dos países analisados.

Embora os fatores na rotação oblíqua apresentem-se correlacionados, essa correlação não contribuiu de forma relevante para obter-se uma estrutura mais simples de cargas para os itens capacidade para o trabalho em relação às exigências físicas do trabalho e prognóstico próprio sobre a capacidade para o trabalho daqui a dois anos. Esses itens permanecem com cargas de valor semelhante e maiores do que 0,30 nos dois fatores em ambas as rotações. Segundo Nunnaly \& Bernstein ${ }^{30}$, as soluções oblíquas e ortogonais tendem a apresentar resultados semelhantes.

Procurando melhor interpretar os resultados obtidos no presente estudo, optou-se por manter o item capacidade para o trabalho em relação às exigências físicas do trabalho no fator I, pois o mesmo forma junto com o item capacidade para o trabalho em relação às exigências mentais do trabalho a segunda dimensão (capacidade para o trabalho em relação às exigências do trabalho) do construto capacidade para o trabalho. O item prognóstico próprio sobre a capacidade para o trabalho daqui a dois anos no instrumento origi- nalmente proposto, não se agrega a outra questão. Além disso, os valores pouco usuais (1, 4 e 7) para as respostas nesse item podem ter contribuído para sua baixa correlação com alguns dos outros da escala. A correlação do mesmo variou de 0,22 ( $\mathrm{p} \leq 0,05$ ) com o item faltas ao trabalho por doenças nos últimos 12 meses até 0,41 ( $\mathrm{p} \leq 0,05$ ) com o item perda estimada para o trabalho devido às doenças. Apesar de no estudo de Martinez et al. 8 o item prognóstico próprio sobre a capacidade para o trabalho daqui a dois anos agruparse ao fator III (presença de doenças e restrições decorrentes do estado de saúde), optou-se por mantê-lo no fator I, uma vez que o presente estudo apresenta uma estrutura semelhante ao das enfermeiras de Radkiewicz et al. ${ }^{23}$, e este item, segundo os autores, apresenta maior grau de subjetividade na sua resposta. Além disso, a inclusão dos itens 2 (capacidade para o trabalho em relação às exigências físicas do trabalho) $\mathrm{e} 7$ (prognóstico próprio sobre a capacidade para o trabalho daqui a dois anos) no fator I reflete um valor maior do alfa $(0,740)$ do que sem estes itens inclusos $(0,673)$, o que corrobora com a decisão de considerar os itens 2 e 7 como componentes do fator I.

Neste estudo, a avaliação dimensional mostrou que os itens que compõem o ICT se agruparam em dois fatores concordando com os aspectos teóricos do construto "capacidade para o trabalho". O primeiro fator pode ser interpretado como a percepção da capacidade para o trabalho e recursos mentais, enquanto o segundo poderia ser nomeado como a presença de doenças e restrições decorrentes do estado de saúde. O número relativamente reduzido de dimensões identificadas nos estudos sobre dimensionalidade do ICT está coerente com a designação de índice dada a esta medida, sugerindo a ideia de uma estrutura mais simples.

Os melhores resultados de confiabilidade do ICT avaliada pelo estimador ômega $(\omega t)$ de McDonald em comparação com o alfa de Cronbach, tanto em relação ao escore global do ICT quanto quando o item é excluído, confirmam o estudo de Revelle e Zinbarg ${ }^{9}$, no qual o ômega de McDonald apresentou valores mais elevados do que o alfa de Cronbrach.

Como o alfa é o menor valor de confiabilidade e o ômega o maior que se pode obter, e considerando ainda que estas são medidas viesadas de confiabilidade, comparar um valor com o outro e também com o maior valor da confiabilidade que se pode obter, a unidade, é uma forma de avaliar o viés cometido ao se utilizar uma ou outra medida. Neste estudo, os valores muito próximos e elevados indicam boa confiabilidade pelos dois indicadores. 
O ICT apresentou maior consistência interna nesta pesquisa $(0,80$ - alfa e 0,87 - ômega) quando comparado aos valores de alfa igual a 0,72 obtidos tanto por Martinez et al. ${ }^{8}$ como por Radkiewicz et al. ${ }^{23}$. Tendo em vista que o grau de correlação dos itens da escala é um fator que influencia a estimativa do alfa, os valores maiores de consistência interna deste estudo podem ser explicados, em parte, pela utilização da medida de correlação para o cálculo do mesmo, em que nesta pesquisa optou-se por utilizar as correlações biserial e policórica, recomendadas para estimar correlação entre variáveis contínuas e ordinais, e entre duas variáveis ordinais, respectivamente. O coeficiente de Pearson subestima a correlação nessa situação.

Componentes principais e eixos principais (principal eixos factoring) são métodos frequentemente utilizados em avaliação dimensional de escalas. Neste estudo, os dois métodos apontaram a mesma estrutura dimensional, embora com cargas e, consequentemente, comunalidades de magnitudes diferentes, tendo em vista que componentes principais baseiam-se na variância total e eixos principais na variância comum.
Recomendações quanto às suposições de cada método e aplicabilidade na psicometria têm sido feitas 19,21 .

Destaca-se na presente pesquisa a utilização do ômega de McDonald como estimador de confiabilidade. Não foram identificados no Brasil estudos utilizando esse estimador, sendo seu uso fora do país ainda reduzido.

Dentre as limitações do presente estudo, ressaltamos que o caráter multidimensional de variáveis associadas ao ICT pode não ter sido contemplado nas correlações apresentadas. Outras variáveis e construtos poderiam estar associados ao ICT e não foram avaliados, o que pode explicar em parte as correlações identificadas que variaram de fraca à moderada. Pesquisas adicionais que complementem a avaliação psicométrica do ICT se fazem necessárias, incluindo avaliações das validades discriminativa e preditiva em grupos ocupacionais brasileiros.

Os resultados em relação à validade e à confiabilidade do ICT dão suporte adicional para a aplicabilidade do índice em pesquisas na área de saúde ocupacional.

\section{Resumo}

Este artigo estima a validade e a confiabilidade do índice de capacidade para o trabalho (ICT) em 1.436 trabalhadores de enfermagem de três hospitais públicos do Rio de Janeiro, Brasil. Foi estimada a validade dimensional, por meio de análise fatorial exploratória e também a validade correlacional, relacionando o ICT com construtos teoricamente relevantes. A confiabilidade foi avaliada pela análise de consistência interna utilizando o coeficiente alfa de Cronbach e ômega de McDonald. A análise fatorial indicou uma estrutura bidimensional, explicando 52,8\% da variância acumulada para os componentes principais e $42 \%$ para os eixos principais. As hipóteses teóricas da validade de construto foram confirmadas com correlação direta e significativa do ICT com os escores de recompensa, controle e autoavaliação do estado de saúde; correlação inversa e significativa com a escala de necessidade de recuperação após o trabalho, distúrbios psíquicos menores, esforço, excesso de comprometimento e demanda. Concluiu-se que o ICT apresentou propriedades psicométricas adequadas.

Pessoa de Saúde; Reprodutibilidade dos Testes; Validade dos Testes 


\section{Colaboradores}

Todos os autores e coautores participaram da concepção e interpretação dos dados, da revisão crítica relevante do conteúdo intelectual e aprovaram a versão final a ser publicada.

\section{Referências}

1. Tuomi K, Ilmarinen J, Jahkola A, Katajarinne L, Tulkki A. Índice de capacidade para o trabalho. São Carlos: EdUFSCar; 2005.

2. Tuomi K, Huuhtanen P, Nykyri E, Ilmarinen J. Promotion of work ability, the quality of work and retirement. Occup Med (Lond) 2001; 51:318-24.

3. Welch LS. Improving work ability in construction workers - let's get to work. Scand J Work Environ Health 2009; 35:321-4.

4. Fischer FM, Borges FN, Rotenberg L, Latorre MR, Soares NS, Rosa PL, et al. Work ability of health care shift workers: what matters? Chronobiol Int 2006; 23:1165-79.

5. Walsh IAP, Corral S, Franco RN, Canetti EEF, Alem MER, Coury HJCG. Capacidade para o trabalho em indivíduos com lesões músculo-esqueléticas crônicas. Rev Saúde Pública 2004; 38:149-56.

6. Martinez MC, Latorre MRDO, Fischer FM. Capacidade para o trabalho: revisão de literatura. Ciênc Saúde Coletiva 2010; 15 Suppl 1:1553-61.

7. Renosto A, Biz P, Hennington EA, Pattussi MC. Confiabilidade teste-reteste do índice de capacidade para o trabalho em trabalhadores metalúrgicos do Sul do Brasil. Rev Bras Epidemiol 2009; 12:217-25.
8. Martinez MC, Latorre MRDO, Fischer FM. Validade e confiabilidade da versão brasileira do índice de capacidade para o trabalho. Rev Saúde Pública 2009; 43:525-32.

9. Revelle W, Zinbarg RE. Coefficients alpha, beta, ômega, and the GLB: comments on Sijtsma. Psychometrika 2009; 74:145-54.

10. Huysamen GK. Coefficient alpha: unnecessarily y ambiguous; unduly y ubiquitous. Journal of Industrial Psychology 2006; 32:34-40.

11. Bentler P, Woodward J. Inequalities among lower bounds to reliability: with applications to test construction and factor analysis. Psychometrika 1980; 45:249-67.

12. Karasek RA. Job Demand, job decision latitude, and mental strain: implications for job redesign. Admin Sci Q 1979; 24:285-308.

13. Siegrist J. Adverse health effects of high-effort/lowreward conditions. J Occup Health Psychocol 1996; 1:27-41.

14. Sluiter JK, van der Beek AJ, Frings-Dresen MHW. The influence of work characteristics on the need for recovery and experienced health: a study on coach drivers. Ergonomics 1999; 42:573-83. 
15. Mari JJ, Williams P. A validity study of a psychiatric screening questionnaire (SRQ-20) in primary care in the city of São Paulo. Br J. Psychiatry 1986; 148:23-6.

16. Rohmert W, Rutenfranz J, editors. Praktische arbeitsphysiologie. Stuttgart/New York: Georg Thieme Verlag; 1983.

17. Tabachnick B, Fidell L. Using multivariate statistics. New York: Harper Collins; 2001.

18. Horn JL. A rationale and test for the number of factors in factor analysis. Psychometrika 1965; 32: 179-85.

19. Fabrigar LR, Wegener DT, MacCallum RC, Strahan, EJ. Evaluation the use of exploratory factor analysis in psychological research. Psychological Methods 1999; 4:272-99.

20. Hair JF, Anderson RE, Tatham RL, Black WC. Análise multivariada de dados. 5a Ed. Porto Alegre: Editora Artmed; 2005.

21. Norris M, Lecavalier L. Evaluating the use of exploratory factor analysis in developmental disability psychological research. J Autism Dev Disord 2010; 40:8-20.

22. Sijtsma K. On the use, the misuse, and the very limited usefulness of Cronbach's alpha. Psychometrika 2009; 74:107-20.

23. Radkiewicz P, Widerszal-Bazyl M; the NEXT-Study Group. Psychometric properties of work ability index in the light of comparative survey study. International Congress Series 2005; 1280:304-9.

24. Ilmarinen J. Work ability: a comprehensive concept for occupational health research and prevention. Scand J Work Environ Health 2009; 35:1-5.

25. Tsutsumi A, Kawakami N. A review of empirical studies on the model of effort-reward imbalance at work: reducing occupational stress by implementing a new theory. Soc Sci Med 2004; 59:2335-59.

26. Kivimaki M, Virtanen M, Elovainio M, Kouvonen A, Vaananen A, Vahtera J. Work stress in the etiology of coronary heart disease: a meta-analysis. Scand J Work Environ Health 2006; 32:431-42.
27. Sanderson K, Andrews G. Common mental disorders in the workforce: recent findings from descriptive and social epidemiology. Can J Psychiatry 2006; 51:63-75.

28. Bethge M, Radoschewski FM, Müller-Fahrnow W. Work stress and work ability: cross-sectional findings from the German sociomedical panel of employees. Disabil Rehabil 2009; 31:1692-9.

29. Sluiter JK, de Croon EM, Meijman TF, Frings-Dresen MHW. Need for recovery from work related fatigue and its role in the development and prediction of subjective health complaints. Occup Environ Med 2003; 60 Suppl 1:i62-70.

30. Nunnaly JC, Bernstein IJ. Teoría psicométrica. Buenos Aires: McGraw Hill; 1995.

31. Chor D, Werneck GL, Faerstein E, Alves MGM, Rotenberg L. The Brazilian version of the effort-reward imbalance questionnaire to assess job stress. Cad Saúde Pública 2008; 24:219-24.

32. Alves MGM, Chor D, Faerstein E, Lopes CS, Werneck GL. Versão resumida da "job stress scale": adaptação para o português. Rev Saúde Pública 2004; 38:164-71.

33. van Veldhoven M, Broersen S. Measurement quality and validity of the need for recovery scale. Occup Environ Med 2003; 60 Suppl 1:i3-9.

34. McCallum J, Shadbolt B, Wang D. Self-rated health and survival: a seven-year follow-up study of Australian elderly. Am J Public Health 1994; 84:1100-5.

35. Idler EL, Kasl SV, Lemke JH. Self-evaluated health and mortality among the elderly in New Haven, Connecticut, and Iowa and Washington counties, Iowa, 1982-1986. Am J Epidemiol 1990; 131: 91-103.

36. Harding TW, Arango MV, Baltazar J, Climent CE, Ibrahim HHA, Ignacio LL, et al. Mental disorders in primary health care: a study of their frequency and diagnosis in four developing countries. Psychol Med 1980; 10:231-41.

Recebido em 14/Out/2010

Versão final reapresentada em 18/Mar/2011

Aprovado em 05/Abr/2011 